\title{
Recurrence of gastric cancer in the jejunal pouch after completion gastrectomy
}

\author{
Tsutomu Namikawa, Michiya Kobayashi, Ken Okamoto, Takehiro Okabayashi, Toyokazu Akimori, \\ Takeki Sugimoto, and Kazuhiro Hanazaki \\ Department of Tumor Surgery, Kochi Medical School, Kohasu-Okocho, Nankoku, Kochi 783-8505, Japan
}

\begin{abstract}
We herein present a case of recurrence of gastric cancer in the jejunal pouch after total gastrectomy in a 74-year-old man. He had a history of two operations for gastric cancer. The second operation was a completion gastrectomy with jejunal pouch reconstruction and regional lymphadenectomy, for gastric cancer in the cardia of the remnant stomach, performed 2 years and 9 months before the present admission. A followup endoscopy showed three elevated tumors along the suture lines in the jejunal pouch in the upper digestive tract. Resection of the jejunal pouch was performed. Gross pathological examination revealed elevated lesions along the staple suture lines in the jejunal pouch. Histopathologically, moderately differentiated tubular adenocarcinoma involving the muscular layer, without lymphatic metastases, was recognized. Recurrence of gastric cancer in the jejunal pouch after resection is rare. We suggest that implantation of exfoliated cancer cells gave rise to the recurrence of tumors on the suture line in this patient. We also review two cases of gastric cancer in the jejunal pouch after resection previously described in the literature.
\end{abstract}

Key words Recurrence $\cdot$ Suture line $\cdot$ Jejunal pouch $\cdot$ Gastric cancer

\section{Introduction}

Although anastomotic recurrence is occasionally encountered after colorectal cancer surgery [1, 2], suture-line recurrence after gastrectomy is rare [3]. We report a case of recurrence of gastric cancer in the jejunal pouch after completion gastrectomy for stump carcinoma.

Offprint requests to: T. Namikawa

Received: November 15, 2006 / Accepted: October 28, 2007

\section{Case report}

A 74-year-old man with a history of two operations for gastric cancer was admitted to our hospital in January 2005. The first operation was a distal gastrectomy and Billroth I reconstruction for early gastric cancer, in 1994. Histologically, the tumor was signet-ring cell carcinoma confined to the submucosa, and no lymph node metastasis was detected.

In January, 2002, an ulcerative tumor, measuring 5.0 $\times 2.5 \mathrm{~cm}$, was found in the lesser curvature of the subcardia in the remnant stomach, and he underwent a completion gastrectomy with regional lymphadenectomy. Reconstruction was by the Roux-en-Y method, with a jejunal pouch. The pouch was created by a making a side-to-side anastomosis of the jejunal loop with a linear stapler and apical section of unstapled jejunal loop, then an esophagojejunostomy was performed using a circular stapler. Histologically, the tumor was moderately differentiated tubular adenocarcinoma invading the proper muscle layer (T2a), without permeation of the lymphatic or venous capillaries. The cancer-stroma relationship was medullary. The proximal and distal resection margins were free of cancer invasion. No lymph node metastasis was seen (stage IB), and the patient was followed up without adjuvant chemotherapy.

In October 2004, a follow-up endoscopy revealed irregularly shaped elevated lesions on the esophagojejunostomy line and along the suture line in the jejunal pouch (Fig. 1). Biopsy showed moderately differentiated tubular adenocarcinoma. Computed tomography showed no lymphadenopathy or hepatic metastasis. The patient's performance status was good, and we decided to perform a third operation with the diagnosis of local recurrence.

In January 2005, we performed a transabdominal resection of the lower esophagus and jejunal pouch. There was no evidence of hepatic or peritoneal recur- 

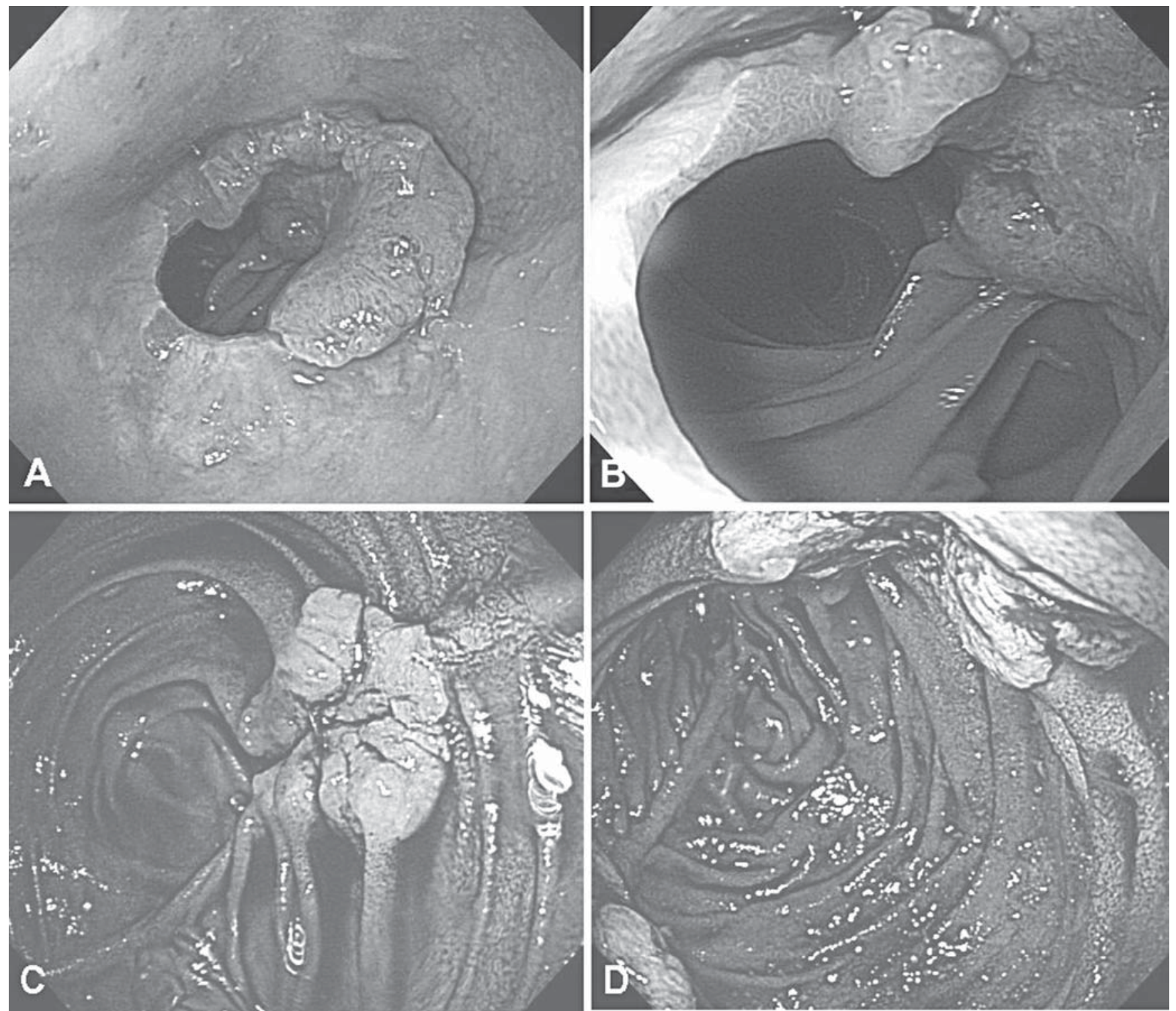

Fig. 1A-D. Endoscopic images, showing irregularly shaped elevated tumors on the anastomotic line of the esophagojejunostomy $(\mathbf{A}, \mathbf{B})$ and along the suture line in the jejunal pouch $(\mathbf{C}, \mathbf{D})$

rence, and the operation was deemed curative. Reconstruction was again by a Roux-en-Y method.

Macroscopically, three separate lesions were evident: a $3.7 \times 2.3 \mathrm{~cm}$ elevated lesion on the esophagojejunostomy line, and two elevated lesions, measuring $8.0 \times$ $2.4 \mathrm{~cm}$ and $3.0 \times 2.4 \mathrm{~cm}$, on the staple suture lines in the jejunal pouch (Fig. 2).

Histologically, the lesions were all moderately differentiated tubular adenocarcinomas invading the proper muscle layer. The cancer-stroma relationship was medullary, with limited involvement of the stroma (Fig. 3). Mild permeation was observed in lymphatic and venous capillaries, but the resected lymph nodes were all negative for cancer.
The postoperative course was uneventful, and the patient shows no evidence of recurrence 2 years and 9 months after the last surgery.

\section{Discussion}

The incidence of anastomotic or suture-line recurrence after gastrectomy is reported to be $3 \%-10 \%$ [3], and the majority of these recurrences are seen on the site of the esophagojejunostomy [4]. Suture-line recurrence in the jejunal pouch is extremely rare. Possible underlying mechanisms include submucosal or subserosal lymphatic spread of cancer, and the implantation of exfoliated cancer cells $[5,6]$. 


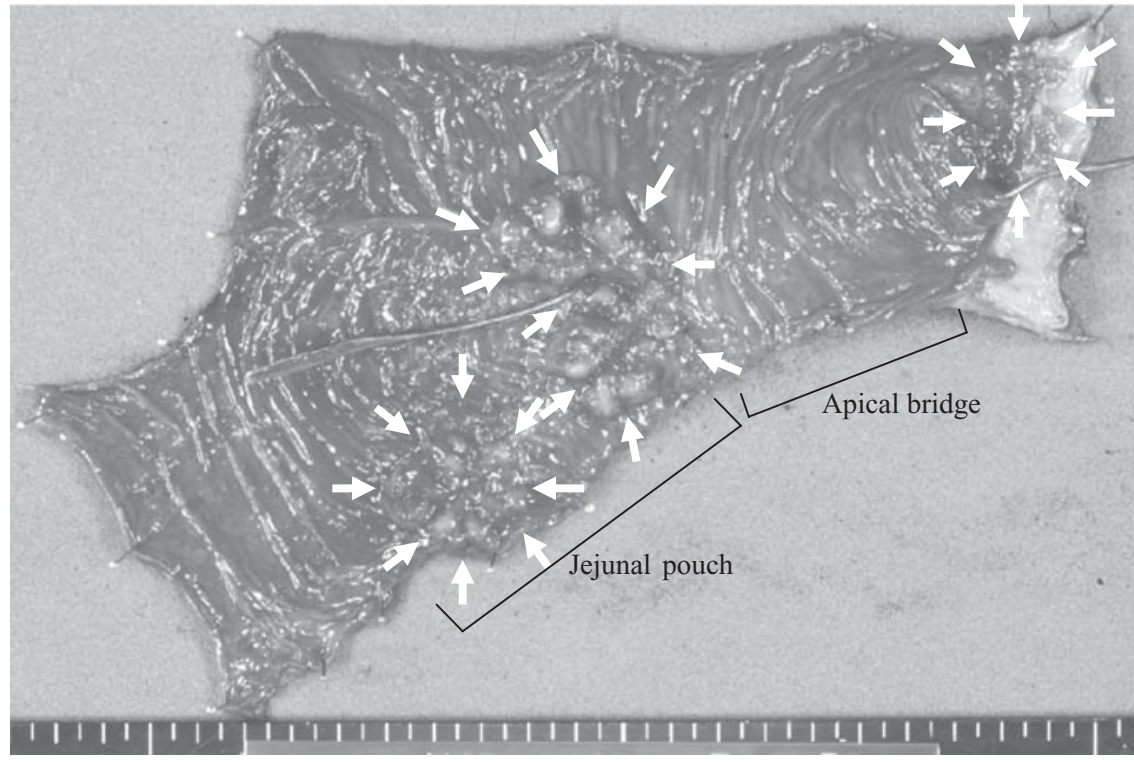

Fig. 2. Surgically resected specimen reveals three elevated tumors (arrows) with central depression - two along the staple suture lines in the jejunal pouch $(8.0 \times 2.4 \mathrm{~cm}$ and $3.0 \times 2.4 \mathrm{~cm}$ in size $)$, and one on the anastomotic line of the esophagojejunostomy $(3.7 \times 2.3 \mathrm{~cm}$ in size)
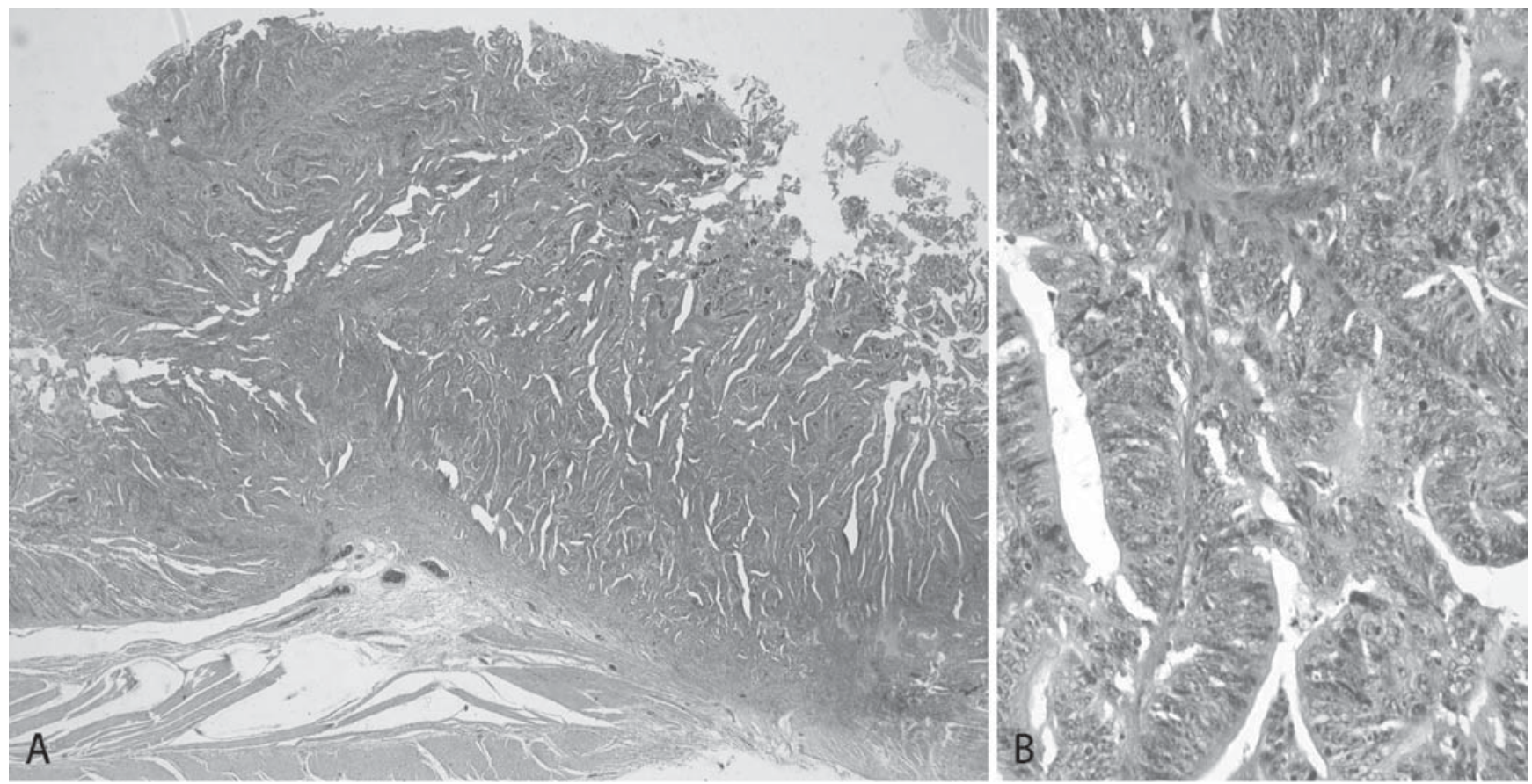

Fig. 3A,B. Histopathological findings of resected specimen of elevated tumor reveal A moderately differentiated tubular adenocarcinoma invading the muscular layer and $\mathbf{B}$ most of the cancer cells showing medullary proliferation, with limited stromal involvement. $\mathrm{H} \& \mathrm{E}, \mathbf{A} \times 40, \mathbf{B} \times 100$

In our patient, lymphatic spread was unlikely to have been the route of recurrence, because the histology of the primary tumor was differentiated type and no lymphatic permeation or lymph node metastasis was seen. The resection line was also negative. The recurrent tumors in the jejunal pouch were elevated mucosal lesions and the depth of invasion was limited to the proper muscle layer, without lesions in the subserosa or outside the serosa.

The implantation of exfoliated cancer cells seems likely to have been the mechanism of recurrence in this patient. It is possible that viable exfoliated cells were implanted in the traumatized cut ends of the suture line. 
Table 1. Reported cases of recurrence of gastric cancer in jejunal pouch after gastrectomy

\begin{tabular}{llll}
\hline Patient no. & 1 & 2 & 3 \\
Author & Miyoshi [7] & Nishimura [8] & Our patient \\
Year & 1999 & 2003 & 2005 \\
Age (years) & 74 & 57 & 74 \\
Sex & $\mathrm{M}$ & $\mathrm{F}$ & $\mathrm{M}$ \\
Interval to recurrence & 4 months & 3 years & 2 years, 9 months \\
Therapy & Observation & Operation & Operation \\
Gross appearance & Elevated & Elevated & Elevated \\
Histological type & Well differentiated & Poorly differentiated (solid) & Moderately differentiated \\
Cancer-Stroma relationship & Medullary & Medullary & Medullary \\
Depth of invasion & Unknown & T3 & T2a \\
Lymph node metastasis & Unknown & Positive & Negative \\
Outcome & Died & Alive & Alive
\end{tabular}

We found two reports of jejunal pouch suture-line recurrence after gastrectomy in Medline and the Japan Centra Revuo Medicina [7, 8]. The clinicopathologic features of these patients, together with those in our patient, are summarized in Table 1. Although the histological types of the tumors differed, the cancer-stroma relationship was medullary in all these patients. Stromal involvement is usually limited when the cancer-stroma relationship is medullary, and the tumor is fragile and can easily break down. This may explain the possibility of implantation of exfoliated cancer cells.

Suture-line recurrence is more frequently encountered in colorectal cancers, but the exact mechanism has not yet been elucidated. As malignant cells adhering to stapling devices are a possible cause of sutureline recurrence [9], thorough lavage of the remnant rectum is recommended [10]. However, a prospective study has denied the effectiveness of this procedure [11]. The host immune response may also play a role. Localized tissue trauma such as the creation of a suture line may give rise to the suppression of local immune function [12]. Furthermore, clots generated in traumatized tissue of suture-line are rich in fibrin, and this fibrin gel may trap exfoliated cancer cells [13].

Although suture-line recurrence after gastrectomy is rare and its mechanism is unclear, we may be able to prevent it by eliminating exfoliated cancer cells, particularly when the use of automatic stapling instruments is involved. We recommend lavage of the jejunum that is to be used for pouch formation and lavage of the esophageal stump prior to anastomosis.

\section{References}

1. Umpleby HC, Williamson RC. Anastomotic recurrence in large bowel cancer. Br J Surg 1987;74:873-8.
2. Southwick HW, Harridge WH, Cole WH. Recurrence at the suture line following resection for carcinoma of the colon. Am J Surg 1962;103:86-9.

3. Papachristou DN, Karas M, Fortner JG. Anastomotic recurrence in the oesophagus complicating gastrectomy for adenocarcinoma of the stomach. Br J Surg 1979;66:609-12.

4. Sugano M, Tsurumaru M, Kajiyama Y, Hashimoto T, Nobukawa B, Hayashida Y. A case of successfully resected stomal recurrence of gastric cancer developed 13 years after total gastrectomy (in Japanese). Nippon Rinsyogeka Gakkai Zasshi (J Jpn Surg Assoc) 2004;65:2913-7.

5. Umpleby HC, Fermor B, Symes MO, Willamson RC. Viability of exfoliated colorectal carcinoma cells. Br J Surg 1984;71:659-63.

6. Skipper D, Cooper AJ, Marston JE, Taylor I. Exfoliated cells and in vitro growth in colorectal cancer. Br J Surg 1987;74:1049-52.

7. Miyoshi K, Fuchimoto S, Ohsaki T, Sakata T, Takeda I, Takahashi K, et al. Suture line recurrence in jejunal pouch replaced after total gastrectomy for gastric cancer. Gastric Cancer 1999:2:194-7.

8. Nishimura M, Honda I, Watanabe S, Nagata M, Souda H, Miyazaki M. Recurrence in jejunal pouch after proximal gastrectomy for early upper gastric cancer. Gastric Cancer 2003;6: 197-201.

9. Gertsch P, Baer HU, Kraft R, Maddern GJ, Altermatt HJ. Malignant cells are collected on circular staplers. Dis Colon Rectum 1992;35:238-41.

10. Long RTL, Edwards RH. Implantation metastasis as a cause of local recurrence of colorectal carcinoma. Am J Surg 1989;157: 194-201.

11. Terzi c, Unek T, Sagol O, Yilmaz T, Fuzun M, Sokmen S, et al. Is rectal washout necessary in anterior resection for rectal cancer? A prospective clinical study. World J Surg 2006, 30:233-41.

12. Curet MJ. Port site metastases. Am J Surg 2004;187:705-12.

13. Jacquet P, Stuart OA, Dalton R, Chang D, Sugarbaker PH. Effect of intraperitoneal chemotherapy and fibrinolytic therapy on tumor implantation in wound sites. J Surg Oncol 1996;62: 128-34. 